\title{
Consensus of Multiagent Systems Described by Various Noninteger Derivatives
}

\author{
G. Nava-Antonio $\mathbb{D}$, ${ }^{1}$ G. Fernández-Anaya, ${ }^{1}$ E. G. Hernández-Martínez $\mathbb{D}{ }^{2}{ }^{2}$ \\ J. J. Flores-Godoy $\mathbb{D},^{3}$ and E. D. Ferreira-Vázquez $\mathbb{D}^{4}$ \\ ${ }^{1}$ Departamento de Física y Matemáticas, Universidad Iberoamericana, México City, Mexico \\ ${ }^{2}$ Departamento de Estudios en Ingeniería para la Innovación, Universidad Iberoamericana, México City, Mexico \\ ${ }^{3}$ Departamento de Matemática, Universidad Católica del Uruguay, Montevideo, Uruguay \\ ${ }^{4}$ Departamento de Ingeniería Eléctrica, Universidad Católica del Uruguay, Montevideo, Uruguay
}

Correspondence should be addressed to J. J. Flores-Godoy; jose.flores@ucu.edu.uy

Received 5 November 2018; Accepted 6 January 2019; Published 26 February 2019

Guest Editor: Carlos-Arturo Loredo-Villalobos

Copyright (C) 2019 G. Nava-Antonio et al. This is an open access article distributed under the Creative Commons Attribution License, which permits unrestricted use, distribution, and reproduction in any medium, provided the original work is properly cited.

In this paper, we unify and extend recent developments in Lyapunov stability theory to present techniques to determine the asymptotic stability of six types of fractional dynamical systems. These differ by being modeled with one of the following fractional derivatives: the Caputo derivative, the Caputo distributed order derivative, the variable order derivative, the conformable derivative, the local fractional derivative, or the distributed order conformable derivative (the latter defined in this work). Additionally, we apply these results to study the consensus of a fractional multiagent system, considering all of the aforementioned fractional operators. Our analysis covers multiagent systems with linear and nonlinear dynamics, affected by bounded external disturbances and described by fixed directed graphs. Lastly, examples, which are solved analytically and numerically, are presented to validate our contributions.

\section{Introduction}

The concept of fractional calculus arose more than three centuries ago, thanks to a question posed by L'Hôpital to Leibniz where the meaning of derivatives of order $1 / 2$ was asked [1]. However, this discipline has gained popularity only in the last decades, in which new methods to solve and analyze fractional differential equations have appeared and researchers have made great efforts to study real phenomena using these tools. This modern boom has occurred mainly because of the capability of fractional order calculus to model certain systems more accurately in comparison with traditional integer order calculus. This greater precision is due to the liberty that fractional calculus gives us to consider noninteger orders for the differential and integral operators.

Throughout the life of this branch of mathematics, various definitions for the fractional derivative have been proposed. A survey of the most common of these can be found in Kilbas, Srivastava, and Trujillo [2]; Petráš [3]; Podlubny [4], along with a rich overview of interesting applications and simulation techniques. In this paper, we will focus on six different fractional derivatives. The first of them is the Caputo fractional derivative which is widely studied in the already-mentioned references and is preferred by many because the Caputo derivative of a constant is zero (which is not true for all fractional derivatives) and the initial conditions of a Caputo fractional system have the same physical interpretation as the integer order case.

The rest of the fractional derivatives addressed in this work are of more recent origin. In the fractional variableorder (also known as time-varying order) derivative, introduced in Samko and Ross [5], the orders of differentiation can be functions of the independent variable or even of other parameters. In Sun, Chen, Wei, and Chen [6] it is argued 
that variable-order calculus allows to better describe certain systems with memory properties that change, for example, with time or position.

The Caputo distributed order derivative, originally presented in Caputo [7], acquired relevance in problems with ultra-slow diffusion, where it has been applied with physical justification, for instance, in Chechkin, Klafter, and Sokolov [8]; Naber [9]. Regarding its meaning, a possible conceptual interpretation for this fractional derivative is suggested in Lorenzo and Hartley [10]: in systems where nonhomogenous or anisotropic properties are involved, it might be appropriate to consider that each differential element of the system should have its own differentiation order.

The fractional derivatives discussed in the above lines do not satisfy the product or the chain rules. Furthermore, the monotonicity of a function is not specified by the sign of those derivatives. The conformable derivative emerged in response to these inconveniences, as explained in Khalil, Al Horani, Yousef, and Sababheh [11]; nevertheless, this fractional operator, which is defined as a limit, loses the memory properties and global character of the others, which are built with integrals. A very similar but more general fractional derivative was proposed in Almeida, Guzowska, and Odzijewicz [12]. That operator is characterized by a kernel function that can be tuned to better represent a given physical system. In the same spirit of the Caputo distributed derivative, in this article we introduce the distributed conformable derivative, a further generalization of the fractional differential operator defined in Khalil et al. [11].

The broad variety of applications that the aforementioned fractional derivatives have had is remarkable: from quantum mechanics, Laskin [13], to control theory, Baleanu, Machado, and Luo [14], and study of human memory and emotions, Tabatabaei, Yazdanpanah, Jafari, and Sprott [15]. In general, these and other problems involving differential equations of fractional order are complicated to approach and in many cases there are no analytical or numerical schemes to solve them. Consequently, the qualitative theory of fractional dynamical systems has become an important line of research. Within this field, the Lyapunov direct method is a tool that allows us to determine the stability and long-term behavior of a certain system, without the need of solving it.

In recent years, Lyapunov stability theory has integrated to fractional calculus; see $\mathrm{Li}$, Chen, and Podlubny [16]; Souahi, Makhlouf, and Hammami [17]; Tabatabaei, Talebi, and Tavakoli [18]; Taghavian and Tavazoei [19]; Wang and $\mathrm{Li}[20]$. One of the objectives of the present article is to take advantage of the similarities between those papers and unify them into a generalized fractional Lyapunov method, useful for systems of differential equations with the fractional derivatives mentioned in the above paragraphs. As an application of this- and with the purpose of comparing the performance of different fractional derivatives in the same problem- we also study in this article the consensus problem of a generalized fractional multiagent system.

A multiagent system is an arrangement of various agents that are organized to accomplish group objectives by means of their local interactions. A multiagent system is said to achieve consensus when the dynamics of the agents converge to a certain desired value. We can appreciate the relevance of this concept by noticing its multiple applications, including: the study of the formation of multivehicle systems [21], the synchronization of coupled oscillators [22], or the distributed sensor fusion in sensor networks [23].

The consensus of multiagent systems has been mostly investigated under the framework of integer order calculus. Extensive introductory reviews of this topic can be found in W. Ren and Beard [21]; W. Ren and Cao [24]. Some of the ideas presented in those references have been generalized for fractional order systems, solely using the Caputo fractional derivative, for example: in $\mathrm{Yu}$, Jiang, $\mathrm{Hu}$, and $\mathrm{Yu}$ [25], an adaptive pinning control is used to realize leader-following consensus in a fractional multiagent system; Yin, Yue, and $\mathrm{Hu}$ [26] studied the consensus problem for fractional heterogeneous systems, made up of agents with different dynamics; Song, Cao, and Liu [27] proposed a distributed protocol to accomplish robust consensus, based on the information of second-order neighbors; Nava-Antonio et al. [28] present sufficient conditions for consensus of multiagent systems with distributed fractional order; and G. Ren and $\mathrm{Yu}$ [29] gave conditions for fractional multiagent systems to achieve robust consensus, via Mittag-Leffler stability methods. That last article is the main inspiration of the second half of this paper, where we will extend the results of G. Ren and Yu [29] to be used in multiagent systems with five other fractional differentiation orders, with linear or nonlinear dynamics, and in the presence of external perturbations.

The order of this text is described next. In Section 2, fundamental preliminary concepts are introduced. Section 3 contains, in two parts, our main results: firstly, we present the generalized fractional Lyapunov direct method and, then, we apply it to study the consensus of multiagent systems modeled with different fractional derivatives. Afterwards, Section 4 gives examples where we verify the validity of the developed theory. Lastly, Section 5 contains the conclusions of the present work.

\section{Preliminary Concepts}

In the following section, we present the definitions of various fractional derivatives and discuss certain properties of systems of equations with these operators. All the definitions below are given considering orders of differentiation $\alpha \in$ $(0,1)$.

Definition 1 (Aguila-Camacho, Duarte-Mermoud, and Gallegos [30]). The Caputo fractional derivative of order $\alpha$ is defined as

$$
T_{1}^{\alpha} x(t)=\frac{1}{\Gamma(1-\alpha)} \int_{t_{0}}^{t} \frac{x^{\prime}(\tau)}{(t-\tau)^{\alpha}} d \tau, \quad t>t_{0}
$$

where $x^{\prime}(t)$ is the integer derivative of $x(t)$. We suppose that $x(t)$ is a differentiable function for all $t \geq t_{0}$ and for all operators in this work. 
Definition 2 (Tabatabaei et al. [18]). The modified initialized Caputo fractional derivative of time-varying order $\alpha(t)$ is defined as follows:

$$
T_{2}^{\alpha} x(t)=\frac{1}{\Gamma(1-\alpha(t))} \int_{0}^{t} \frac{x^{\prime}(\zeta)}{(t-\zeta)^{\alpha(t)}} d \zeta+\Psi_{c}^{x}(t),
$$

$\forall t \geq 0$,

where $\Psi_{c}^{x}(t)=(1 / \Gamma(1-\alpha(t))) \int_{-c}^{0}(t-\zeta)^{-\alpha(t)} x^{\prime}(\zeta) d \zeta$ captures the behavior of $x$ before $t=0$, assuming that $x$ begins from $-c<0$. Since we will focus in this paper on systems which are at rest at $t<0, \Psi_{c}^{x}(t)=0$.

Definition 3 (Jiao, Chen, and Podlubny [31]). The distributed order fractional derivative in the Caputo sense with respect to the density function $c:[\mu, 1] \longrightarrow[0,+\infty)$ for some $1>$ $\mu>0$, such that $\int_{\mu}^{1} c(\alpha) d \alpha \neq 0$, is defined as follows:

$$
T_{3}^{\alpha} x(t)=\int_{\mu}^{1} c(\alpha) T_{1}^{\alpha} x(t) d \alpha .
$$

The Laplace transform of a distributed order derivative, which will appear in the derivation of our main results, is

$$
\mathscr{L}\left\{T_{3}^{\alpha} x(t)\right\}=C(s) X(s)-\frac{1}{s} C(s) x\left(0^{+}\right),
$$

where $C(s)=\int_{m-1}^{m} c(\alpha) s^{\alpha} d \alpha$.

Definition 4 (Souahi et al. [17]). The conformable fractional derivative starting from $a$ of a function $x$ defined on $[a, \infty)$ is

$$
T_{4}^{\alpha} x(t)=\lim _{\epsilon \longrightarrow 0} \frac{x\left(t+\epsilon(t-a)^{1-\alpha}\right)-x(t)}{\epsilon},
$$

for all $t>a$. If $\lim _{t \rightarrow a^{+}} T_{4}^{\alpha} x(t)$ exists, then

$$
T_{4}^{\alpha} x(a)=\lim _{t \rightarrow a^{+}} T_{4}^{\alpha} x(t) .
$$

Definition 5 (Almeida et al. [12]). Let $k:[a, b] \longrightarrow \mathbb{R}$ be a continuous nonnegative map such that $k(t) \neq 0$, whenever $t>a$ and $x:[a, b] \longrightarrow \mathbb{R}$. By definition, $x$ is $\alpha$-differentiable at $t>a$, with respect to kernel $k$, if the limit

$$
T_{5}^{\alpha} x(t)=\lim _{\epsilon \rightarrow 0} \frac{x\left(t+\epsilon k(t)^{1-\alpha}\right)-x(t)}{\epsilon}
$$

exists. The local fractional derivative at $t=a$ is defined by

$$
T_{5}^{\alpha} x(a)=\lim _{t \rightarrow a^{+}} T_{5}^{\alpha} x(t),
$$

if the limit exists.

Definition 6. Let $k$ and $x$ be functions as in Definition 5 and $c$ a density function as in Definition 3. The distributed conformable fractional derivative is defined as

$$
T_{6}^{\alpha} x(t)=\int_{0}^{1} c(\alpha) T_{5}^{\alpha} x(t) d \alpha .
$$

Theorem 7 (Almeida et al. [12]). A function $x:[a, b] \longrightarrow \mathbb{R}$ is $\alpha$-differentiable at $t>a$ if and only if it is differentiable at $t$. In that case, we have the relation

$$
T_{5}^{\alpha} x(t)=k(t)^{1-\alpha} x^{\prime}(t), \quad t>a .
$$

Notice that Definition 5 is a particular case of Definition 6 . Then by Theorem 7 if we take $k(t)=t-a$ we obtain

$$
T_{4}^{\alpha} x(t)=(t-a)^{1-\alpha} x^{\prime}(t), \quad t>a .
$$

In a similar form by substituting (10) in (9) we have

$$
T_{6}^{\alpha} x(t)=x^{\prime}(t) \int_{0}^{1} c(\alpha) k(t)^{1-\alpha} d \alpha .
$$

Consider the generalized system of fractional differential equations of order $\alpha \in(0,1)$ :

$$
T_{j}^{\alpha} x=f(t, x(t)), \quad j=1,2,3,4,5, \text { or } 6,
$$

where $x \in \mathbb{R}^{n}, x\left(t_{0}\right)=x_{0}$, and $f: \mathbb{R}_{+} \times \mathbb{R}^{n} \longrightarrow$ $\mathbb{R}^{n}$ is a given nonlinear function is Lipzchitz with respect to the second argument. For simplicity and without loss of generality, we will consider that the equilibrium points of the systems analyzed hereafter are at the origin, i.e., $f(t, 0)=0$, $\forall t \geq 0$.

Throughout this paper, we will assume that the studied systems have unique solutions. The existence and uniqueness of the solution of system (13) is discussed in Podlubny [4], Xu and $\mathrm{He}$ [32], Ford and Morgado [33], and Bayour and Torres [34], for the cases $j=1,2,3$, and 4 , respectively. The theory of existence and uniqueness of solutions when $j=5$ or 6 can be easily generalized from Bayour and Torres [34], by taking into account (11) and (12).

The Final Value Theorem and an important Laplace transform associated with fractional calculus, both used in the following sections of this article, are presented next.

Theorem 8 (Duffy [35]). Let $F(s)=\mathscr{L}\{f(t)\}$. If all poles of $s F(s)$ are in the open left-half complex plane, then

$$
\lim _{t \rightarrow \infty} f(t)=\lim _{s \rightarrow 0} s F(s) .
$$

Definition 9 (Podlubny [4]). A two-parameter function of the Mittag-Leffler type is defined by

$$
E_{\alpha, \beta}(z)=\sum_{k=0}^{\infty} \frac{z^{k}}{\Gamma(\alpha k+\beta)}, \quad \alpha>0, \beta>0
$$

Lemma 10 (Podlubny [4]). The Mittag-Leffler function of two parameters satisfies the following relationship:

$$
\mathscr{L}^{-1}\left[\frac{s^{-(\alpha-\beta)}}{s^{\beta}-a}\right]=t^{\alpha-1} E_{\beta, \alpha}\left(a t^{\beta}\right), \quad\left|s^{\beta}-a\right|<1 .
$$

\section{Lyapunov Stability for Generalized Fractional Systems}

The two Theorems in this section summarize the known results for Lyapunov stability theory for nonlinear systems 
of (A) Li et al. [16] and Wang and Li [20] (Definition 1); (B) Souahi et al. [17] (Definition 2); (C) Tabatabaei et al. [18] (Definition 3); (D) Taghavian and Tavazoei [19] (Definition 4); (E) Almeida et al. [12] (Definition 5) and also extends the Lyapunov stability theory for nonlinear systems defined by operators introduced in Definitions 5 and 6. Specifically, in (A) the Lyapunov direct method for standard Caputo fractional system (13) with $j=1$ is proved and the definition of Mittag-Leffler stability is introduced. In (B) the same result for the case of the modified initialized Caputo fractional derivative of time-varying order $\alpha(t)$ with $j=2$ is proved. For the case of distributed fractional systems (13) the mentioned result in (C) for $j=3$ is proved. In the case of conformal fractional systems in (D), it is shown that (13) is fractional exponentially stable which implies asymptotic stability for $j=4$. For the case of Definitions 5 and 6 we show that the proofs are very similar to the one for the case $j=4$. In consequence, Theorems 12 and 16 extend the Lyapunov direct method for generalized fractional systems defined in (13).

Assumption 11. For $j=2$, system (13) is autonomous, i.e., $f(t, x(t))=f(x(t))$.

Theorem 12. Consider system (13) with $j=1,2,3,4,5$ or 6. Let $V(t, x(t))$ be a continuously differentiable function such that

$$
\begin{aligned}
\gamma_{1}\|x(t)\|^{l} & \leq V(t, x(t)) \leq \gamma_{2}\|x(t)\|^{l m}, \\
T_{j}^{\alpha} V(t, x(t)) & \leq-\gamma_{3}\|x(t)\|^{l m},
\end{aligned}
$$

where $t \geq 0, \gamma_{i}(i=1,2,3)$, and $l$ and $m$ are arbitrary positive constants. If Assumption 11 is fulfilled, then the origin of system (13) is asymptotically stable.

Proof.

(i) For $j=1,(13)$ is a standard Caputo fractional system. For this system, the proof is the same as the one of Theorem 5.1 of Li et al. [16]. There, it is shown that (13) is Mittag-Leffler stable, which implies asymptotic stability.

(ii) For $j=2$, (13) is a fractional system of timevarying order. This proof follows from Theorem 1 of Tabatabaei et al. [18]. That result requires the weaker hypotheses $V(t, x(t)) \geq 0, V(t, x(t))=0 \Longleftrightarrow x=0$ (which are implied by (17)) and $T_{2}^{\alpha} V(t, x(t))<0$ in $D-\{0\}$ (which is implied by (18)).

(iii) For $j=3$, (13) is a distributed fractional system. In this case, the proof can be found in Theorem 4.1 of Taghavian and Tavazoei [19].

(iv) For $j=4,(13)$ is a conformable fractional system. This proof is the same as the one of Theorem 1 of Souahi et al. [17], where it is shown that (13) is fractional exponentially stable. That kind of stability also implies asymptotic stability.

(v) For $j=5$ or 6 , (13) is a system with local fractional derivatives or a distributed conformable fractional system, respectively. In these instances, the proofs are very similar to the one of the previous case $(j=4)$. That proof depends on two facts about the conformable derivative: that it satisfies the product rule in the traditional sense and that the sign of $T_{4}^{\alpha} x(t)$ determines the monotonicity of $x(t)$. Note, from (8) and (12), that these features are also true for the operators $T_{5}^{\alpha}$ and $T_{6}^{\alpha}$.

The next result is a partial generalization of Theorem 12, being more permissive with the Lyapunov function and its fractional derivative, but requiring a couple of additional hypotheses.

Assumption 13. For $j=3$ in (13), the Lyapunov function of Theorem 16 has a nonzero initial value, i.e., $V(0, x(0)) \neq 0$.

Definition 14 (Teel and Praly [36]). A function $h:[0, \infty) \longrightarrow$ $[0, \infty)$ is said to belong to class $\mathscr{K}$ if it is continuous, zero at zero, and strictly increasing.

Assumption 15. For $j=4,5$, and 6 in (13), the class $\mathscr{K}$ functions $h_{i}(i=1,2,3)$ satisfy $\lim _{t \rightarrow \infty} h_{i}(t)=\infty$.

Theorem 16. Consider system (13) with $j=1,2,3,4,5$ or 6. Suppose that there exist class $\mathscr{K}$ functions $h_{i}(i=1,2,3)$ and $a$ continuously differentiable function $V(t, x(t))$ such that

$$
\begin{aligned}
h_{1}(\|x(t)\|) & \leq V(t, x(t)) \leq h_{2}(\|x(t)\|), \\
T_{j}^{\alpha} V(t, x(t)) & \leq-h_{3}(\|x(t)\|) .
\end{aligned}
$$

If Assumptions 11, 13, and 15 are fulfilled, then the origin of system (13) is asymptotically stable.

Proof.

(i) For $j=1$, the proof can be found on Theorem 6.2 of Li et al. [16].

(ii) For $j=2$, the proof is presented in Tabatabaei et al. [18], as explained in item (ii) of Theorem 12 proof.

(iii) For $j=3$, the proof is the same as the one of Theorem 4.2 of Taghavian and Tavazoei [19].

(iv) For $j=4$, proof can be found in Theorem 3 of Souahi et al. [17].

(v) For $j=5$ or 6 , considering the argument stated in (v) of Theorem 12 proof, we can readily generalize the result of $j=4$ to cases of the distributed conformable and local fractional derivatives.

We now know that Theorem 16 is valid also for RiemannLiouville-like fractional difference equations (see Theorem 3.6 in $\mathrm{Wu}$, Baleanu, and Luo [37]). So we conjecture that Theorem 16 can be valid for a larger family of operators.

The following lemma contains a property of the generalized fractional differential operator which is useful when putting into practice the previous Lyapunov Stability Theorems. 
Lemma 17. Let $x: \mathbb{R} \longrightarrow \mathbb{R}$ be a continuous differentiable function. Then, for $j=1,2,3,4,5$ or 6 , the following relationship holds:

$$
\frac{1}{2} T_{j}^{\alpha}\left[x^{T}(t) P x(t)\right] \leq x^{T}(t) P T_{j}^{\alpha}[x(t)],
$$

where $P$ is a Hermitian positive definite matrix.

Proof. The proof for the cases $j=1,2,4$ can be found in Aguila-Camacho et al. [30]; Souahi et al. [17]; Tabatabaei et al. [18], respectively. If $j=3$, a proof for when $P=$ $I$ is presented in Fernández-Anaya, Nava-Antonio, JamousGalante, Muñoz-Vega, and Hernández-Martínez [38]. To obtain the more general version, consider inequality (21) with $j=1$, multiply it by the distribution function $c(\alpha) \geq 0$, and integrate

$$
\begin{aligned}
\int_{0}^{1} & \frac{1}{2} c(\alpha) T_{1}^{\alpha}\left[x^{T}(t) P x(t)\right] d \alpha=\frac{1}{2} T_{3}^{\alpha}\left[x^{T}(t) P x(t)\right] \\
& \leq \ldots \\
\ldots & \leq \int_{0}^{1} x^{T}(t) P c(\alpha) T_{1}^{\alpha}[x(t)] d \alpha \\
& =x^{T}(t) P T_{3}^{\alpha}[x(t)] .
\end{aligned}
$$

We can follow a similar reasoning to prove this lemma for $j=5$ or 6 , by multiplying (21) with $j=4$ and $\alpha=$ 1 (that is, the traditional integer order derivative) by $k(t)^{1-\alpha}$ or $\int_{0}^{1} c(\alpha) k(t)^{1-\alpha} d \alpha$ and using properties (8) or (12), respectively.

\section{Application to the Consensus of Multiagent Systems of Generalized Fractional Order}

In this section, we will investigate the problem of consensus for generalized multiagent systems. First, we will consider systems with nonlinear dynamics and then we will present the linear simplification of that analysis.

4.1. Graph Theory Fundamentals. We can describe the interaction topology of a multagent system with the help of graph theory. A graph $\mathscr{G}$ is characterized by its vertices $\mathscr{V}=$ $\left\{v_{1}, v_{2}, \ldots, v_{n}\right\}$ (which represent the agents of the system) and its edges $\mathscr{W} \subseteq \mathscr{V}^{2}$ (which correspond to the agents' relationships). In this paper, we will focus on directed graphs, where each edge is an ordered pair $\left(v_{i}, v_{j}\right)$; this means that agent $j$ receives information from agent $i$. A graph can be represented by its adjacency matrix $A=\left[a_{i j}\right] \in \mathbb{R}^{n \times n}$, where $a_{i j}=1$ if $\left(v_{i}, v_{j}\right) \in \mathscr{W}$ and $a_{i j}=0$ if $\left(v_{i}, v_{j}\right) \notin \mathscr{W}$, or by its Laplacian matrix $L=\left[l_{i j}\right] \in \mathbb{R}^{n \times n}$, where $l_{i i}=\sum_{j \in N_{i}} a_{i j}$ and $l_{i j}=-a_{i j}$ for $i \neq j$ with $N_{i}$ the number of connected nodes to node $i$.

The following lemmas will be used in the proofs of our main results to gain insight into the graphs associated with the multiagent systems of our interest.
Lemma 18 (W. Ren and Cao [24]). If a graph has a directed spanning tree, then the Laplacian matrix L has a simple zero eigenvalue and all its other eigenvalues have positive real parts. Moreover, all eigenvalues of $H=L+B$ will have positive real parts, where $B=\operatorname{diag}\left\{b_{1}, b_{2}, \ldots, b_{n}\right\}$ and $b_{i} \geq 0$ is not all 0 .

Lemma 19 (Zhang and Tian [39]). Let $E=\left[1_{n-1},-I_{n-1}\right] \epsilon$ $\mathbb{R}^{(n-1) \times n}$ and $F=\left(\begin{array}{c}0_{n-1}^{T} \\ -I_{n-1}\end{array}\right) \in \mathbb{R}^{n \times(n-1)}$, where $1_{n-1}$ is the column vector of ones, $I_{n-1}$ is the identity matrix, and $0_{n-1}$ is the zero column vector, and each of them is of size $n-1$. Then, $C=-E L F$ is Hurwitz, where $L$ is the Laplacian matrix, if and only if the associated interaction graph has a directed spanning tree.

The notion of consensus that will be considered throughout this paper is presented next.

Definition 20. A multiagent system accomplishes consensus if it fulfills the following condition:

$$
\begin{aligned}
& \lim _{t \rightarrow \infty}\left\|x_{i}(t)-x_{k}(t)\right\| \leq 0, \\
& \forall i, k \in\{1,2, \ldots, n\}, i \neq k,
\end{aligned}
$$

where $x_{k}(t)$ is the state of the $k$-th agent.

Hereinafter, we will suppose, for simplicity, that all agents are in a one-dimensional space. All our results can be easily generalized for $m$ dimensions by means of the Kronecker product. Moreover, in this work we will consider the matrix norm:

$$
\|A\|=\sqrt{\sum_{i=1}^{n} \sum_{j=1}^{m} a_{i j}^{2}},
$$

with $A=\left(a_{i j}\right) \in \mathbb{R}^{n \times m}$. And for any matrix $Q \in \mathbb{R}^{n \times n}$, $\lambda_{\text {max }}(Q)$, and $\lambda_{\text {min }}(Q)$ denote the largest and smallest eigenvalues, respectively.

4.2. Robust Consensus of Nonlinear Generalized Fractional Multiagent Systems. A generalized nonlinear fractional multi-agent system can be represented by

$$
\begin{aligned}
T_{j}^{\alpha} x_{i}(t)=f\left(t, x_{i}(t)\right)+u_{i}(t)+w_{i}(t) & \\
& \\
& \quad i \in\{1,2, \ldots, n\},
\end{aligned}
$$

where $j=1,2,3,4,5$ or 6 and $x_{i}(t), f\left(t, x_{i}(t)\right), u_{i}(t)$, and $w_{i}(t)$ are the state, nonlinear dynamics, control input, and external disturbances of the $i$-th agent, respectively.

As an auxiliary element, we will consider a virtual leader, which is an isolated agent that designates objectives for the states of all other agents. The behavior of the virtual leader is characterized by

$$
T_{j}^{\alpha} x_{r}(t)=f\left(t, x_{r}(t)\right)
$$


where $x_{r}(t)$ is the state of the virtual leader. To accomplish consensus in system (25), we will use the following control input:

$$
\begin{aligned}
u_{i} & (t) \\
& =-\beta\left[\sum_{k=1}^{n} a_{i k}\left(x_{i}(t)-x_{k}(t)\right)+b_{i}\left(x_{i}(t)-x_{r}(t)\right)\right],
\end{aligned}
$$

where $a_{i k}$, for $i, k \in\{1,2, \ldots, n\}$ with $i \neq k$, is the $(i, k)$-th entry of the adjacency matrix $A \in \mathbb{R}^{n \times n}$ associated with the undirected graph describing the interaction of the agents, and $\beta \geq 0$ and $b_{i}$, for $(i=1,2, \ldots, n)$, are positive constants to be chosen as mentioned in Theorem 23.

We will require that the following assumptions hold.

Assumption 21. The disturbance signal $w_{i}(t)$ satisfies $\left\|w_{i}(t)\right\| \leq l<\infty \forall i \in\{1,2, \ldots, n\}$.

Assumption 22. For the multiagent system (25) with $j=3$, the distribution function $c(\alpha)$ is such that

$$
\mathscr{L}^{-1}\left\{\frac{1}{C(s)+\mu / \lambda_{\max }(Q)}\right\} \geq 0,
$$

where $C(s)$ is defined in terms of $c(\alpha)$ as in (4).

Theorem 23. Consider the generalized fractional nonlinear multiagent system (25) with the virtual leader (26) and the controller (27). Assume that the nonlinear function $f(t, x(t))$ is Lipschitz (with respect to $x$ and with Lipschitz constant $\theta$ ) and that the associated fixed directed graph has a directed spanning tree.

(1) For $j=1,2,3,4,5$ or 6: if $w_{i}(t)=0, \forall i$, Assumption 11 is satisfied and

$$
\frac{\sqrt{2} \beta}{\theta} \geq\|Q\|
$$

where $Q>0$ is the solution of the Lyapunov equation $H^{T} \mathrm{Q}+\mathrm{QH}=3 I_{n}$, and then robust consensus is achieved.

(2) For $j=1$ or 3 if $\exists w_{i}(t) \neq 0$, Assumptions 21 and 22 are satisfied, and

$$
\frac{\beta}{\theta} \geq\|Q\|,
$$

where $Q>0$ is the solution of the Lyapunov equation $H^{T} Q+Q H=3 I_{n}$, and then the steady-state errors of any two agent will converge as $t \longrightarrow \infty$ to the region $M_{1}$, where

$$
M_{1}=\left\{\left|x_{i}(t)-x_{y}(t)\right| \leq \sqrt{\frac{2 n \lambda_{\text {max }}(Q)}{\beta \mu \lambda_{\text {min }}(Q)}}\|Q\| l\right\},
$$

and $\mu=\beta-\|Q\|^{2} \theta^{2} / \beta$.
Proof. By substituting (27) in system (25), we can write

$$
\begin{aligned}
T_{j}^{\alpha} X(t)= & F(X(t))-\beta\left[L X(t)+B\left(X(t)-x_{r} 1_{n}\right)\right] \\
& +W(t),
\end{aligned}
$$

where $F(X(t))=\left[f\left(x_{1}(t)\right), \ldots, f\left(x_{n}(t)\right)\right]^{T}$. Subtracting $T_{j}^{\alpha}\left[1_{n} x_{r}(t)\right]$ from both sides of (32) and using the change of variables $z_{i}(t)=x_{i}(t)-x_{r}(t), i \in\{1,2, \ldots, n\}$ yields

$$
T_{j}^{\alpha} Z(t)=-\beta H Z(t)+\Delta F(Z(t))+W(t),
$$

where $H$ is defined as in Lemma $18, Z(t)=\left[z_{1}(t), \ldots, z_{n}(t)\right]^{T}$ and $\Delta F(Z(t))=\left[f\left(z_{1}(t)+x_{r}(t)\right)-f\left(x_{r}(t)\right), \ldots, f\left(z_{n}(t)+\right.\right.$ $\left.\left.x_{r}(t)\right)-f\left(x_{r}(t)\right)\right]^{T}$. Consider the following Lyapunov candidate function for system (33):

$$
V(t)=Z^{T} Q Z(t) .
$$

Applying Lemma 17 and substituting (33), we can analyze $T_{j}^{\alpha} V(t)$ :

$$
\begin{aligned}
T_{j}^{\alpha} V(t) \leq & \beta Z^{T}(t)\left[-Q H-H^{T} Q\right] Z(t) \\
& +2 Z^{T}(t)[Q \Delta F(Z(t))+Q W(t)] .
\end{aligned}
$$

Using Lemma 18 we can conclude that all the eigenvalues of $H$ have positive real parts, so that $-H$ is Hurwitz. Thus, there exists a matrix $Q=Q^{T}>0$ that satisfies $-H^{T} Q-Q H=$ $-3 I_{n}$. Applying in (35) this identity along with the property $\xi^{T} \zeta+\zeta^{T} \xi \leq \kappa \xi^{T} \xi+(1 / \kappa) \zeta^{T} \zeta$, which is valid for any $\xi, \zeta \in \mathbb{R}^{m}$, we obtain

$$
\begin{aligned}
T_{j}^{\alpha} V(t) \leq & -3 \beta\|Z(t)\|^{2}+\beta\|Z(t)\|^{2}+\frac{1}{\beta}\|Q W(t)\|^{2} \\
& +\beta\|Z(t)\|^{2}+\frac{1}{\beta}\|Q\|^{2}\|\Delta F(t, Z(t))\|^{2} .
\end{aligned}
$$

Since $f(t, x(t))$ is Lipschitz with respect to $x(t)$, we can simplify (36) as follows:

$$
\begin{aligned}
T_{j}^{\alpha} V(t) & \\
\leq & -\beta\|Z(t)\|^{2}+\frac{n l^{2}\|Q\|^{2}}{\beta} \\
& \quad+\frac{\|Q\|^{2}}{\beta} \sum_{i=1}^{n}\left(f\left(t, z_{i}(t)+x_{r}(t)\right)-f\left(t, x_{r}(t)\right)\right)^{2} \\
\leq & -\beta\|Z(t)\|^{2}+\frac{n l^{2}\|Q\|^{2}}{\beta}+\frac{\|Q\|^{2} \theta^{2}}{\beta}\|Z(t)\|^{2} \\
\leq & -\mu\|Z(t)\|^{2}+\frac{n l^{2}\|Q\|^{2}}{\beta}
\end{aligned}
$$

where $\mu=2 \beta-\|Q\|^{2} \theta^{2} / \beta>0$ by (29).

(1) In the following, we will use Theorem 12 to prove that system (33) is asymptotically stable at its origin. 
If $w_{i}(t)=0, \forall i$, then $l=0$. As consequence, (37) turns into $T_{j}^{\alpha} V(t) \leq-\mu\|Z(t)\|^{2}$, so that (18) is satisfied for $\alpha_{3}=\mu$. Additionally, noting that $\lambda_{\min }(Q) Z^{T}(t) Z(t) \leq V(t) \leq \lambda_{\max }(Q) Z^{T}(t) Z(t)$, it is clear that $V(t)$ satisfies (17) for $\alpha_{1}=\lambda_{\text {min }}(Q)$ and $\alpha_{2}=\lambda_{\max }(Q)$. By Theorem 12 we can conclude that system (33) is asymptotically stable at $Y(t)=$ $0_{n-1}$. This means, according to the definition of $Z(t)$, that $\lim _{t \rightarrow \infty}\left\|x_{1}(t)-x_{i}(t)\right\|=0, \forall i \in\{1,2, \ldots, n\}$, and hence the multiagent system (25) achieves robust consensus.

(2) Using the inequality $Z^{T}(t) P Z(t) \leq \lambda_{\max }(Q)\|Z(t)\|^{2}$ in (34) yields $V(t) / \lambda_{\max }(Q) \leq\|Z(t)\|^{2}$. Hence

$$
T_{j}^{\alpha} V(t) \leq-\frac{\mu}{\lambda_{\max }(Q)} V(t)+\frac{n l^{2}\|Q\|^{2}}{\beta} .
$$

Let $u(t)=V(t)-n l^{2}\|Q\|^{2} \lambda_{\max }(Q) / \mu \beta$. The generalized fractional derivative of $u(t)$ can be analyzed as follows:

$$
\begin{aligned}
T_{j}^{\alpha} u(t) & \leq-\frac{\mu}{\lambda_{\max }(Q)} V(t)+\frac{n l^{2}\|Q\|^{2}}{\beta} \\
& \leq-\frac{\mu}{\lambda_{\max }(Q)} u(t) .
\end{aligned}
$$

There exists a nonnegative function $m(t)$ satisfying

$$
T_{j}^{\alpha} u(t)+m(t)=-\frac{\mu}{\lambda_{\max }(Q)} u(t) .
$$

From this point, we will only consider $j=3$ and then we will obtain the same result for $j=1$ as a particular case. Taking the Laplace transform of (40) produces

$$
B(s)\left[U(s)-\frac{u(0)}{s}\right]+M(s)=-\frac{\mu}{\lambda_{\max }(Q)} U(s),
$$

where $B(s)$ is defined as in (4), and $U(s)$ and $M(s)$ are the Laplace transforms of $u(t)$ and $m(t)$, respectively. Solving for $U(s)$ we obtain

$$
U(s)=\frac{(B(s) / s) u(0)}{B(s)+\mu / \lambda_{\max }(Q)}-\frac{M(s)}{B(s)+\mu / \lambda_{\max }(Q)} .
$$

Note that the inverse Laplace Transform of the second term of the right-hand side of (42) is nonnegative, since $m(t)$, $\mathscr{L}^{-1}\left\{1 /\left(B(s)+\mu / \lambda_{\max }(Q)\right)\right\} \geq 0$. Considering this, we can turn (42) into

$$
u(t) \leq \mathscr{L}^{-1}\left\{\frac{(B(s) / s) u(0)}{B(s)+\mu / \lambda_{\max }(Q)}\right\} .
$$

Substituting the definition of $u(t)$ into (43) yields

$$
\begin{aligned}
V( & (t)-\frac{n l^{2} \lambda_{\max }(Q)\|Q\|^{2}}{\beta \mu} \\
& \leq \mathscr{L}^{-1}\left\{\frac{B(s) u(0)}{s\left(B(s)+\mu / \lambda_{\max }(Q)\right)}\right\} .
\end{aligned}
$$

By using Theorem 8 we can calculate the limit of (44) as $t \longrightarrow$ $\infty$. Note that $\lim _{s \rightarrow 0} B(s)=0$. Then

$$
\begin{gathered}
\lim _{t \rightarrow \infty}\left\{V(t)-\frac{n l^{2} \lambda_{\max }(Q)\|Q\|^{2}}{\beta \mu}\right\} \\
\leq \lim _{s \rightarrow 0} \frac{B(s) u(0)}{B(s)+\beta / \lambda_{\max }(Q)}=0 .
\end{gathered}
$$

Considering that $\lambda_{\min }(Q)\|Z(t)\|^{2} \leq V(t)$, it follows from (45) that

$$
\lim _{t \rightarrow \infty}\|Z(t)\| \leq \frac{\sqrt{n \lambda_{\max }(Q) / \lambda_{\min }(Q)}\|Q\| l}{\sqrt{\beta \mu}} .
$$

According to the definition of $Z(t)$ and using inequality properties, we obtain

$$
\begin{aligned}
\left|x_{i}(t)-x_{y}(t)\right| & \leq\left|x_{r}(t)-x_{i}(t)\right|+\left|x_{r}(t)-x_{y}(t)\right| \\
& \leq\left|z_{i}(t)\right|+\left|z_{y}(t)\right| \\
& \leq \sqrt{2\left(\left|z_{i}(t)\right|^{2}+\left|z_{y}(t)\right|^{2}\right)} \\
& \leq \sqrt{2}\|Z(t)\|,
\end{aligned}
$$

$\forall i, y \in\{1,2, \ldots, n\}$. Combining (46) and (47), we can analyze the limit as $t \longrightarrow \infty$ of the difference between any pair of agents:

$$
\lim _{t \rightarrow \infty}\left|x_{i}(t)-x_{y}(t)\right| \leq \frac{\sqrt{2 n \lambda_{\max }(Q) / \lambda_{\min }(Q)}\|Q\| l}{\sqrt{\beta \mu}}
$$

$\forall i, y \in\{1,2, \ldots, n\}$, which proves that the steady-state errors between the agents converge to $M_{1}$.

We can prove this theorem with $j=1$ by considering the case $j=3$ and setting the distribution function of $T_{3}^{\alpha}$ as $c(\alpha)=$ $\delta(\alpha-a)$, which turns this operator into the standard Caputo fractional derivative of order $a$. Furthermore, notice that

$$
\begin{aligned}
& \mathscr{L}^{-1}\left\{\frac{1}{C(s)+\mu / \lambda_{\max }(Q)}\right\} \\
& =\mathscr{L}^{-1}\left\{\frac{1}{s^{\beta}+\mu / \lambda_{\max }(Q)}\right\} \\
& =t^{\beta-1} E_{\beta, \beta}\left(-\frac{\mu}{\lambda_{\max }(Q)} t^{\beta}\right) \geq 0,
\end{aligned}
$$

where we have used Lemma 10. This means that Assumption 22 is satisfied. Alternatively, the case $j=1$ is derived in Theorem 2 of G. Ren and Yu [29].

4.3. Robust Consensus of Linear of Generalized Fractional Multiagent Systems. A linear generalized fractional multiagent system with external disturbances can be described as a particular case of (25), with $f\left(t, x_{i}\right)=0$ :

$$
T_{i}^{\alpha} x_{i}(t)=u_{i}(t)+w_{i}(t), \quad i \in\{1,2, \ldots, n\},
$$


where $x_{i}(t), u_{i}(t)$, and $w_{i}(t)$ are the state, control input, and external disturbances of the $i$ th agent, respectively.

In order to accomplish robust consensus we can use as simpler controller than (27)

$$
u_{i}(t)=-\beta \sum_{k=1}^{n} a_{i j}\left(x_{i}(t)-x_{k}(t)\right)
$$

where $\beta \geq 0$ and $a_{i k}(i, k=1,2, \ldots, n ; i \neq k)$ is the $(i, k)$ th element of the adjacency matrix $A \in \mathbb{R}^{n \times n}$ associated with the directed graph describing the interaction of the agents. By following a procedure completely analogous to the one done in the previous section, the following theorem can be readily proved.

Theorem 24. Consider the generalized fractional nonlinear multiagent system (50) with the control input (51). Suppose that the associated fixed directed graph has a directed spanning tree.

(1) For $j=1,2,3,4,5$ or 6: if $w_{i}(t)=0, \forall i$, then system (50) achieves robust consensus.

(2) For $j=1$ or 3: if $\exists w_{i}(t) \neq 0$ and Assumptions 11, 21, and 22 are satisfied, then the steady-state errors of any two agents will converge to the region $M_{2}$, defined as

$$
M_{2}=\left\{\left|x_{i}(t)-x_{y}(t)\right| \leq \frac{\sqrt{2 n \lambda_{\max }(P)}\|P E\| l}{\beta \sqrt{\lambda_{\min }(P)}}\right\},
$$

where $\lambda_{\max }(P)$ and $\lambda_{\min }(P)$ are the maximum and minimum eigenvalues of the matrix $P>0$, which is the solution of the Lyapunov equation $C^{T} P+P C=-2 I_{n-1}$, and $E, C$ are defined as in Lemma 19.

\section{Examples}

Example 1. Consider a group of 3 undisturbed agents described by (50) with $w_{i}(t)=0, \forall i$, under the influence of controller (51), with the interaction graph shown in Figure 1. The Laplacian matrix associated with this system is

$$
L=\left[\begin{array}{ccc}
2 & -1 & -1 \\
-1 & 2 & -1 \\
-1 & -1 & 2
\end{array}\right]
$$

From Figure 1, it is clear that this graph has a directed spanning tree. Therefore, by Theorem 24 , this system accomplishes consensus. In order to verify our prediction, we solved this problem for the six types of fractional derivatives addressed in this text. To this end, we considered the initial conditions $x_{1}(0)=0.7996, x_{2}(0)=3.9978, x_{3}(0)=-4.7974$ and the parameter $\beta=1$. Additionally, we used the differentiation orders given in Table 1 .

The cases $j=1$ and $j=2$ were analyzed numerically with the aid of the MATLAB functions developed in Petráš [40] and Valério [41]; Valério, Vinagre, Domingues, and Da Costa [42]. Taking advantage of (10) and (12), the cases $j=4, j=5$, and $j=6$ were worked out with MATLAB's standard ODE Solver. Given the limitations of the existing computational methods to study fractional distributed order equations, we solved the case $j=3$ analytically, as it is shown next.

We can rewrite the system in vector and obtain

$$
T_{3}^{\alpha} X(t)=-\beta L X(t) .
$$

By taking the Laplace transform of (54) and solving for $\mathbb{X}(s)$, we get

$$
\begin{aligned}
\mathbb{X}(s) & =[C(s) I+L]^{-1}\left[\frac{C(s)}{s} X(0)\right] \\
& =\frac{1}{s(B(s)+3)}\left[\begin{array}{l}
B(s) x_{1}(0)+q \\
B(s) x_{2}(0)+q \\
B(s) x_{3}(0)+q
\end{array}\right],
\end{aligned}
$$

where $q=\sum_{i=1}^{3} x_{i}(0)$. Substituting $B(s)=s^{\beta}+4 s^{\beta / 2}$, decomposing the right hand side of (55) into partial fractions, and taking their inverse Laplace transforms yields

$$
X(t)=\left[\begin{array}{l}
x_{1}(0)+\frac{q-3 x_{1}(0)}{2} t^{1 / 2} E_{1 / 2,3 / 2}\left(-t^{1 / 2}\right)+\frac{3 x_{1}(0)-q}{2} t^{1 / 2} E_{1 / 2,3 / 2}\left(-3 t^{1 / 2}\right) \\
x_{2}(0)+\frac{q-3 x_{2}(0)}{2} t^{1 / 2} E_{1 / 2,3 / 2}\left(-t^{1 / 2}\right)+\frac{3 x_{2}(0)-q}{2} t^{1 / 2} E_{1 / 2,3 / 2}\left(-3 t^{1 / 2}\right) \\
x_{3}(0)+\frac{q-3 x_{3}(0)}{2} t^{1 / 2} E_{1 / 2,3 / 2}\left(-t^{1 / 2}\right)+\frac{3 x_{3}(0)-q}{2} t^{1 / 2} E_{1 / 2,3 / 2}\left(-3 t^{1 / 2}\right)
\end{array}\right] .
$$

which are the expressions shown in Figure 4.

In Figures 2-7 we can see the behavior of the error between the states of the multiagents. In all the cases these errors converge to zero as expected and depending on the characteristics of the operator $T_{j}^{\alpha}$ this rate of convergence varies.
Example 2. Consider again system (54), with the same interaction topology as in Example $1, \beta=1$, but this time with the disturbances $w_{i}(t)=\gamma_{i}+a_{i} e^{-c_{i} t}$, where $\gamma_{i}, a_{i}, c_{i} \in \mathbb{R}$, $\forall i \in\{1,2,3\}$. Let the differentiation orders be $\alpha=0.5$ and $c(\alpha)=\delta(\alpha-2 / 3)+4 \delta(\alpha-1 / 3)$ for $j=1$ and $j=3$, respectively. Assumption 21 is fulfilled, since the external disturbances are 


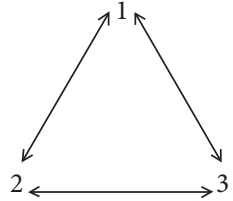

FIGURE 1: Interaction graph for the 3 agents of Examples 1, 2, and 3.

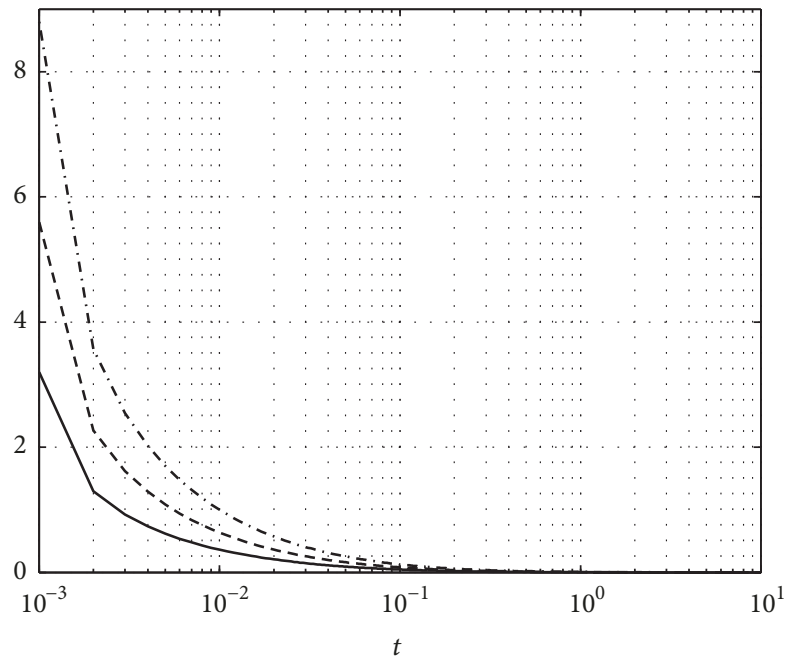

$-\left|x_{1}-x_{2}\right|$
$--\left|x_{2}-x_{3}\right|$
$--\left|x_{2}-x_{3}\right|$

Figure 2: Linear case, $j=1$.

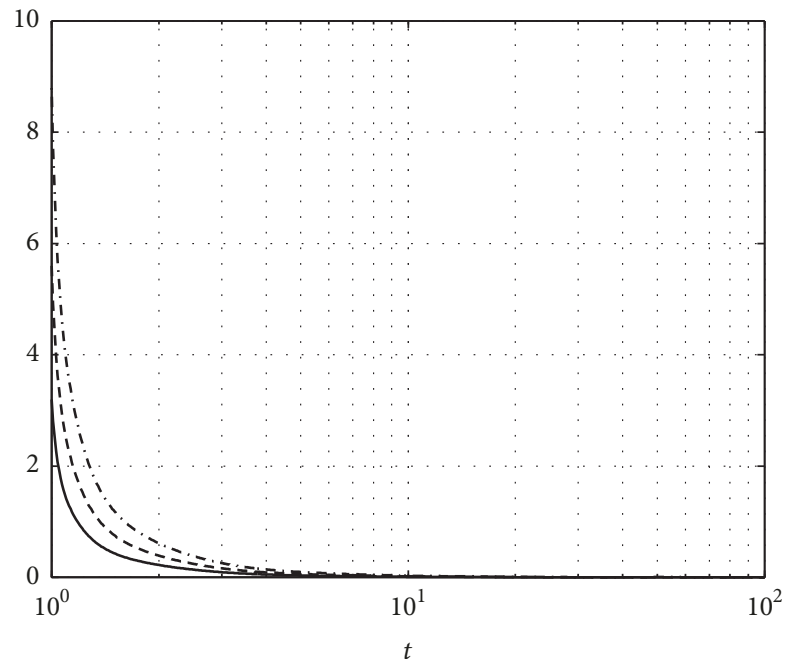

$-\left|x_{1}-x_{2}\right|$

$---\left|x_{2}-x_{3}\right|$

$\cdot \cdot-\left|x_{2}-x_{3}\right|$

FIGURE 3: Linear case, $j=2$.
TABLE 1: Differential orders for simulations.

\begin{tabular}{lc}
\hline$j$ & Parameters \\
\hline 1 & $\alpha=0.5$ \\
2 & $\alpha(t)=1-\frac{\exp (-t / 50)}{2}$ \\
3 & $c(\alpha)=\delta(\alpha-v)+4 \delta\left(\alpha-\frac{\nu}{2}\right), v=\frac{2}{3}$ \\
4 & $a=0, \alpha=0.5$ \\
5 & $k(t)=1+0.4 \log (t+1)$ \\
6 & $c(\alpha)=\delta(\alpha-v)+4 \delta\left(\alpha-\frac{\nu}{2}\right), v=\frac{2}{3}, k(t)=1+0.4 \log (t+1)$ \\
\hline
\end{tabular}

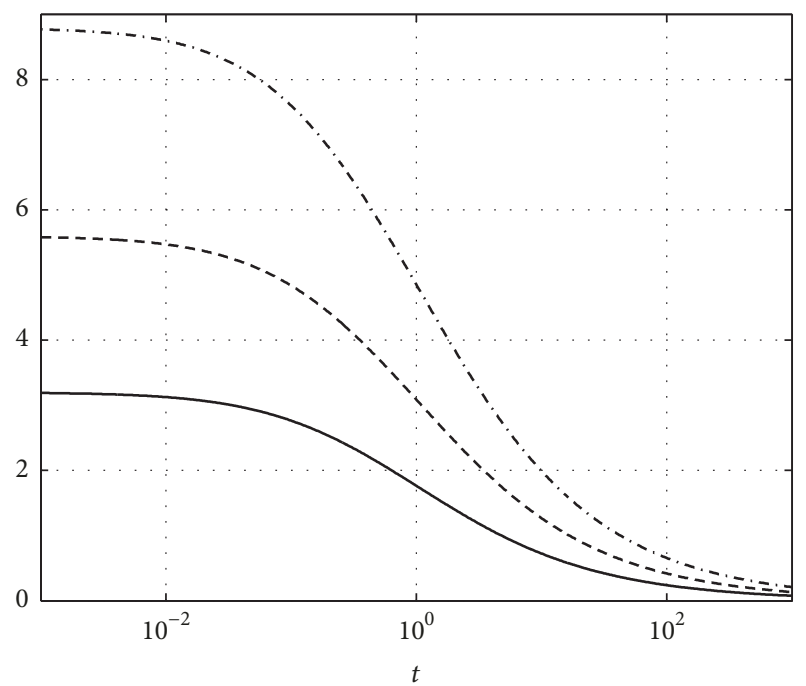

$-\left|x_{1}-x_{2}\right|$
$---\left|x_{2}-x_{3}\right|$
$\ldots-\left|x_{2}-x_{3}\right|$

Figure 4: Linear case, $j=3$.

bounded by $\max _{i \in\{1,2,3\}}\left\{\left\|\gamma_{i}+a_{i}\right\|\right\}$. Hence, we only need to show that Assumption 22 is also satisfied in order to apply Theorem 24. In this specific problem, the left-hand side of (28) is

$$
\begin{aligned}
\mathscr{L}^{-1} & \left\{\frac{1}{C(s)+\beta / \lambda_{\max }(P)}\right\}=\mathscr{L}^{-1}\left\{\frac{1}{s^{2 / 3}+4 s^{1 / 3}+3}\right\} \\
= & \mathscr{L}^{-1}\left\{\frac{1}{s^{1 / 3}+3}\right\} * \mathscr{L}^{-1}\left\{\frac{1}{s^{1 / 3}+1}\right\} \\
= & {\left[t^{-2 / 3} E_{1 / 3,1 / 3}\left(-3 t^{1 / 3}\right)\right] *\left[t^{-2 / 3} E_{1 / 3,1 / 3}\left(-3 t^{1 / 3}\right)\right] } \\
= & \int_{-\infty}^{+\infty}(t-\tau)^{-2 / 3} E_{1 / 3,1 / 3}\left(-3(t-\tau)^{1 / 3}\right) \\
\cdot & t^{-2 / 3} E_{1 / 3,1 / 3}\left(-3 t^{1 / 3}\right) d \tau,
\end{aligned}
$$

where we have used Theorems 8 and 16. Considering that all the factors inside the integral in (57) are nonnegative, we can conclude that Assumption 22 is fulfilled and, therefore, the steady-state errors between the agents will converge asymptotically to $M_{1}$. Solving the equation $C^{T} P+P C=$ 


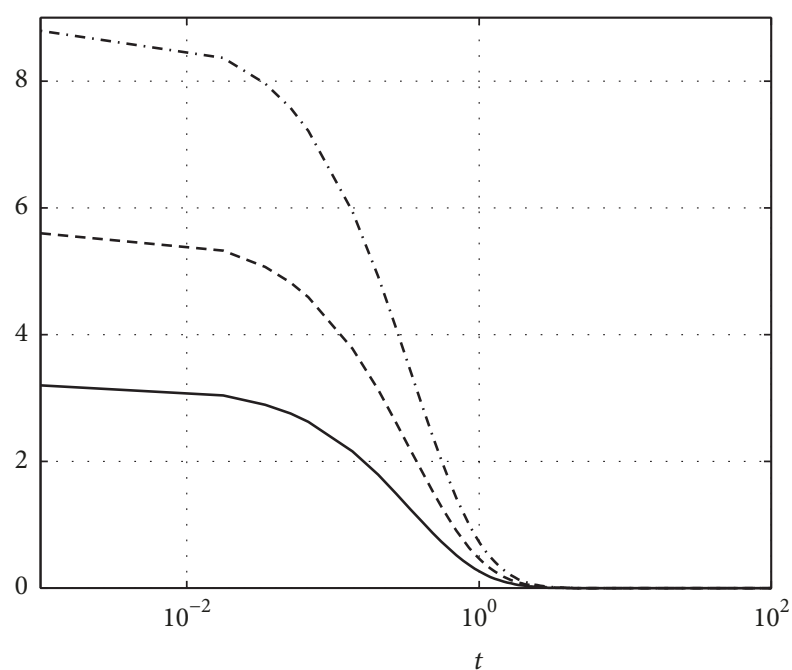

$-\left|x_{1}-x_{2}\right|$
$---\left|x_{2}-x_{3}\right|$
$\cdots-\left|x_{2}-x_{3}\right|$

FIgURE 5: Linear case, $j=4$.

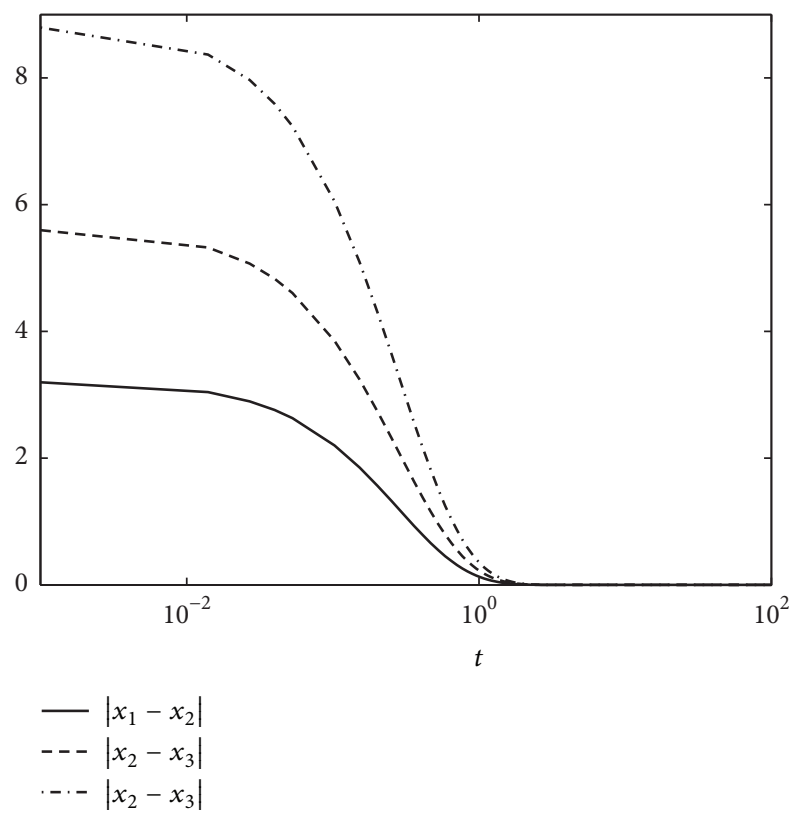

Figure 6: Linear case, $j=5$.

$-2 I_{n-1}$ yields $P=(1 / 3) I$, so that $\lambda_{\max }(P)=\lambda_{\min }(P)=1 / 3$. Moreover, $\|P E\|=\max _{1 \leq j \leq 3}\left\{\sum_{i=1}^{2}\left|(P E)_{i j}\right|\right\}=2 / 3$. By setting the parameters $\gamma_{1}=-2, \gamma_{2}=1, \gamma_{3}=2, a_{1}=1, a_{2}=2$, $a_{3}=-1, c_{1}=2, c_{2}=1.5$, and $c_{3}=1.7$, one can calculate that the disturbances are bounded by $l=3$. Substituting these values in the definition of $M_{1}$ produces

$$
M_{1}=\left\{\left|x_{i}(t)-x_{j}(t)\right| \leq 2 \sqrt{6} \approx 4.8989\right\} .
$$

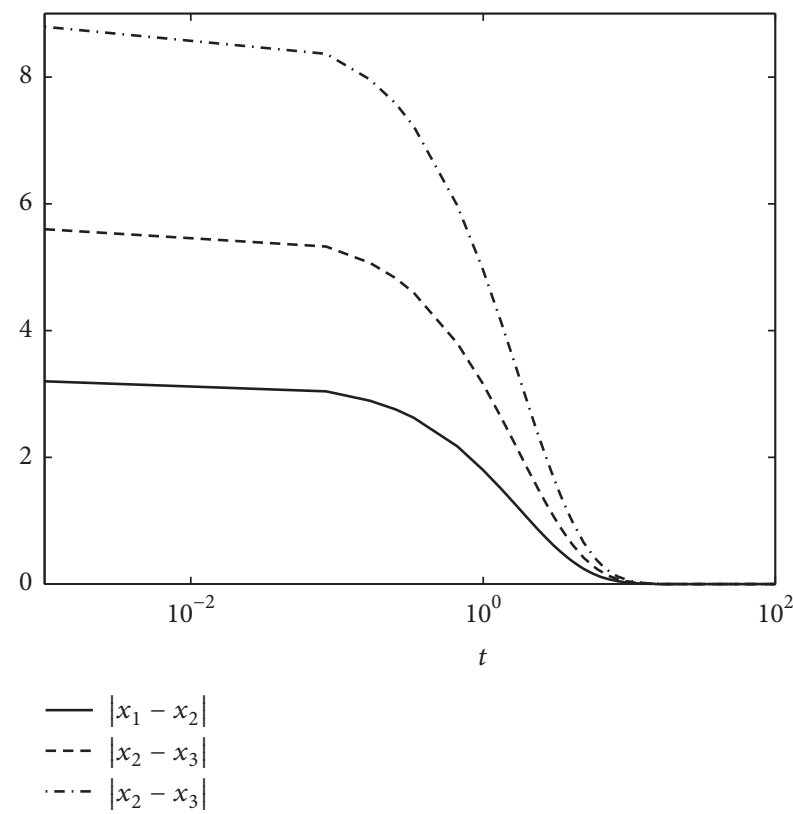

Figure 7: Linear case, $j=6$.

To verify this analysis, we solved this system numerically, for $j=1$ (using the MATLAB functions of Petrás [40]), and analytically, for $j=3$ (since there are no suitable numerical methods). For the case $j=3$, we can take the Laplace transform of (54) and solve for $\mathbb{X}(s)$ :

$$
\begin{aligned}
\mathbb{X}(s)= & {[C(s) I+L]^{-1}\left[\mathbb{W}(s)+\frac{C(s)}{s} X(0)\right] } \\
= & \frac{1}{s C(s)(C(s)+3)} \\
& \times\left[\begin{array}{l}
C(s) s w_{1}(s)+C^{2}(s) x_{1}(0)+s \sum_{i=1}^{3} w_{i}(s) \\
C(s) s w_{2}(s)+C^{2}(s) x_{2}(0)+s \sum_{i=1}^{3} w_{i}(s) \\
C(s) s w_{3}(s)+C^{2}(s) x_{3}(0)+s \sum_{i=1}^{3} w_{i}(s)
\end{array}\right],
\end{aligned}
$$

where $\mathscr{L}^{-1}\{W(t)\}=\mathbb{W}(s)$, and, for simplicity, we have considered $x_{1}(0)+x_{2}(0)+x_{3}(0)=0$. Substituting $C(s)=$ $s^{2 / 3}+4 s^{1 / 3}$ and $w_{i}(s)=\gamma_{i} / s+a_{i} /\left(s+c_{i}\right)$, one can decompose the right hand side of (59) into partial fractions and take their inverse Laplace transforms. After extensive calculations, we obtain

$$
X(t)=G(t)+H(t)+f(t) X(0)
$$


where $f(t), G=\left[g_{1}(t), g_{2}(t), g_{3}(t)\right]^{T}, H=h(t)[1,1,1]^{T}$ are defined as follows:

$$
\begin{aligned}
f( & t)=\frac{12 t^{-1 / 3}}{9 \Gamma(2 / 3)}-\frac{39 t^{-2 / 3}}{27 \Gamma(1 / 3)}+\frac{3 t^{-2 / 3} E_{1 / 3,1 / 3}\left(-t^{1 / 3}\right)}{2} \\
- & \frac{3 t^{-2 / 3} E_{1 / 3,1 / 3}\left(-3 t^{1 / 3}\right)}{54}, \\
g_{i}(t) & =\gamma_{i}\left\{\frac{1}{3}-\frac{4 t^{-1 / 3}}{9 \Gamma(2 / 3)}+\frac{13 t^{-2 / 3}}{27 \Gamma(1 / 3)}\right\} \\
& +\frac{a_{i}}{c_{i}^{2}-28 c_{i}+27}\left\{\left(9+4 c_{i}\right) e^{-c_{i} t}\right. \\
& \left.+13 t^{-2 / 3} E_{1,1 / 3}\left(-c_{i} t\right)-\left(12+c_{i}\right) t^{-1 / 3} E_{1,2 / 3}\left(-c_{i} t\right)\right\} \\
& +t^{-2 / 3}\left[\left(\frac{a_{i}}{2\left(c_{i}-1\right)}-\frac{\gamma_{i}}{2}\right) E_{1 / 3,1 / 3}\left(-t^{1 / 3}\right)\right. \\
& \left.+\left(\frac{\gamma_{i}}{54}-\frac{a_{i}}{2\left(c_{i}-27\right)}\right) E_{1 / 3,1 / 3}\left(-3 t^{1 / 3}\right)\right],
\end{aligned}
$$

$\forall i \in\{1,2,3\}$ and

$$
\begin{aligned}
h(t) & =\sum_{i=1}^{3}\left\{\gamma_{i}\left[\frac{t^{1 / 3}}{12 \Gamma(4 / 3)}-\frac{19}{144}+\frac{265 t^{-1 / 3}}{1728 \Gamma(2 / 3)}\right]\right. \\
& +\frac{t^{-2 / 3}}{\Gamma(1 / 3)}\left(\frac{a_{i}}{12 c_{i}}-\frac{3355 \gamma_{i}}{20736}\right) \\
& +t^{-2 / 3}\left[E_{1 / 3,1 / 3}\left(-t^{1 / 3}\right)\left(\frac{\gamma_{i}}{6}-\frac{a_{i}}{6\left(c_{i}-1\right)}\right)\right. \\
& +E_{1 / 3,1 / 3}\left(-3 t^{1 / 3}\right)\left(\frac{a_{i}}{6\left(c_{i}-27\right)}-\frac{\gamma_{i}}{162}\right) \\
& \left.+E_{1 / 3,1 / 3}\left(-4 t^{1 / 3}\right)\left(\frac{\gamma_{i}}{768}-\frac{a_{i}}{12\left(c_{i}-64\right)}\right)\right] \\
& +\frac{a_{i}}{c_{i}\left(c_{i}^{3}-92 c_{i}^{2}+1819 c_{i}-1728\right)} \times\left[228 c_{i}\right. \\
& +45 c_{i}^{2} e^{-c_{i} t}-\left(8 c_{i}^{2}+265 c_{i}\right) t^{-1 / 3} E_{1,2 / 3}\left(-c_{i} t\right) \\
& \left.\left.+\left(c_{i}^{2}+128 c_{i}+144\right) t^{-2 / 3} E_{1,1 / 3}\left(-c_{i} t\right)\right]\right\} .
\end{aligned}
$$

For both cases $j=1$ and $j=3$, Figure 8 depicts the states of the agents and Figure 9 the errors between them. To plot our results, we used the initial conditions $x_{1}(0)=$ $-30, x_{2}(0)=10, x_{3}(0)=20$. From these figures, we can confirm that the steady-state errors of the agents converge to the calculated region.

Example 3. Consider the nonlinear system described by the interaction graph shown in Figure 1 and (25) and (26), where $f(t, x(t))=\arctan (x(t))$, for which we can take its Lipschitz constant as $\theta=1$. For this system, one can calculate $\|Q\|=$

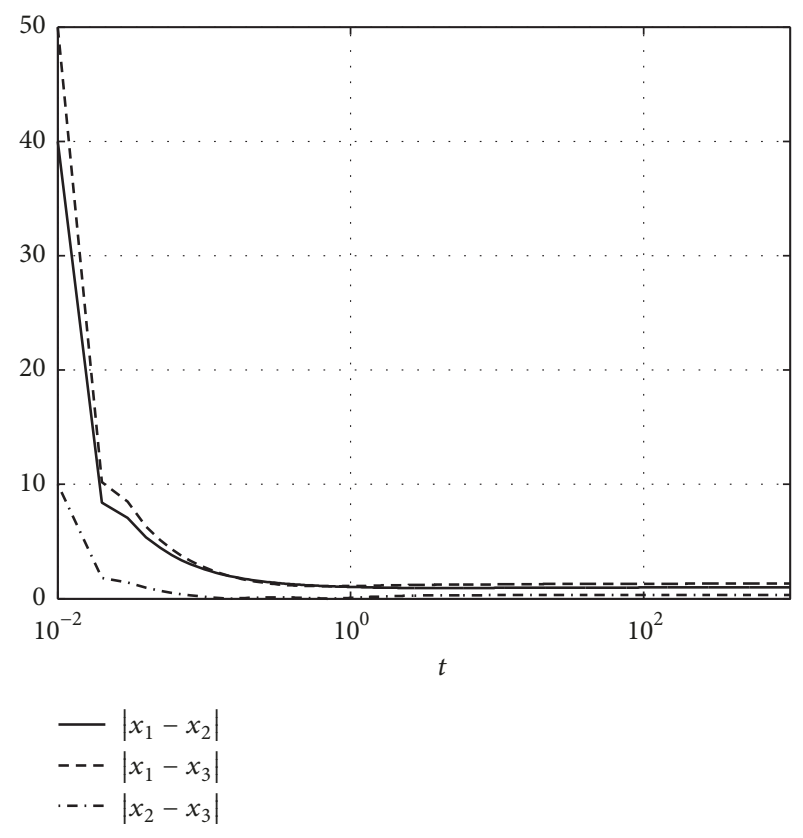

FIGURE 8: Linear case with perturbation, $j=1$.

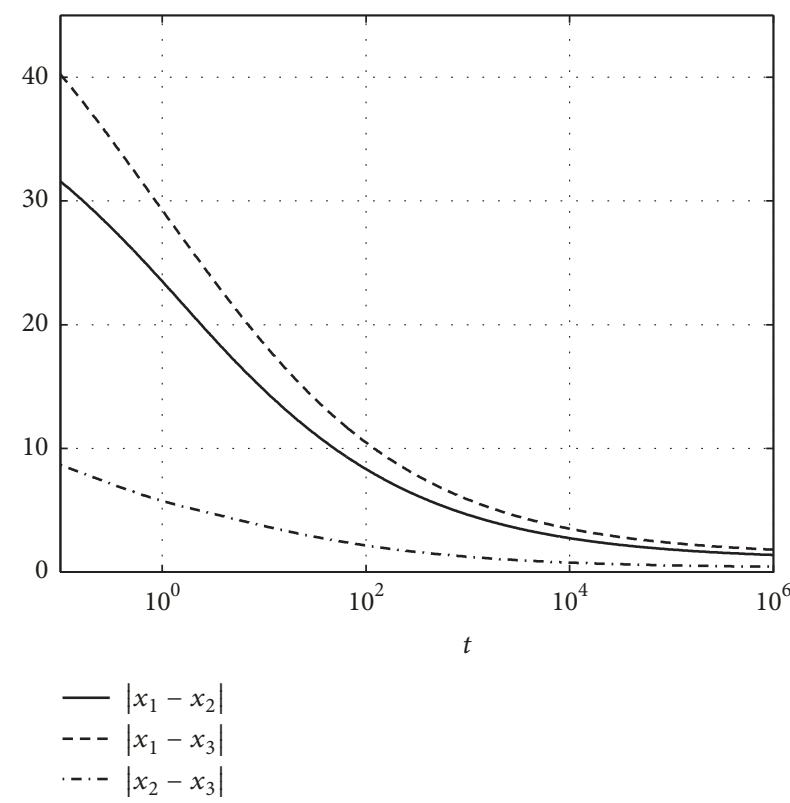

FIGURE 9: Linear case with perturbation, $j=3$.

$3 \sqrt{26} / 23$. Setting the parameters of the controller as $\beta=1$, $b_{1}=1, b_{2}=2$, and $b_{3}=3$ allows us to fulfill inequality (29) and, thus, according to Theorem 23, this system achieves consensus.

All the simulations start with zero initial conditions and constant input such that the agents evolve with different trajectories; at time $t=3$ the agents start using the control law given by (27). The simulation for the different operators are shown in Figures 10-14 where we plotted the errors between states of the different agents for $j=1,2,4,5$, and 6 (see 


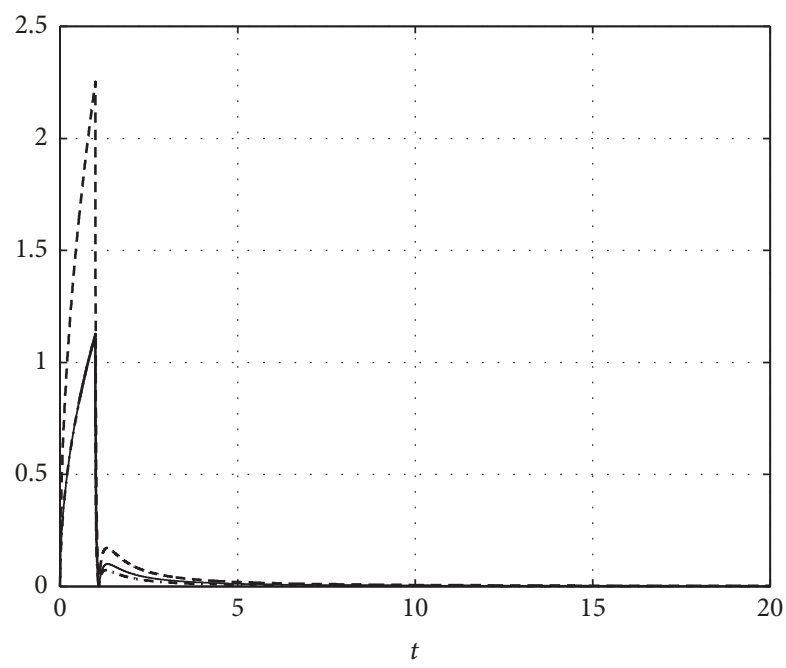

$-\left|x_{1}-x_{2}\right|$
$---\left|x_{2}-x_{3}\right|$
$\cdots-\left|x_{2}-x_{3}\right|$

Figure 10: No-linear case, $j=1$.

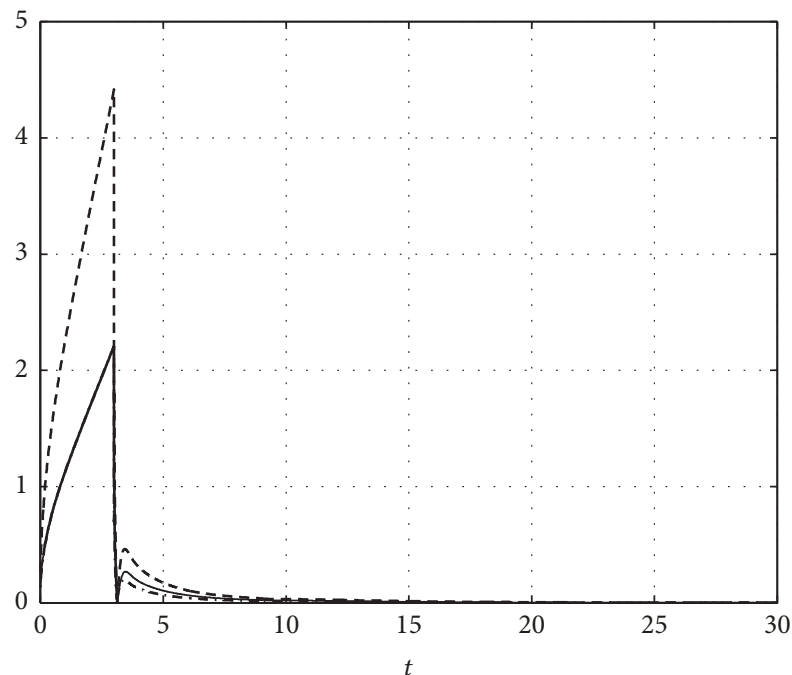

$$
\begin{aligned}
& -\left|x_{1}-x_{2}\right| \\
& ---\left|x_{2}-x_{3}\right| \\
& \cdots-\left|x_{2}-x_{3}\right|
\end{aligned}
$$

FIgURE 11: No-linear case, $j=2$.

Table 1), with the computational tools already mentioned. We do not present the solution of this system for $j=3$ since neither the available numerical methods for distributed order systems nor the Laplace transform technique used in the previous examples are applicable for the nonlinear case.

In all the simulation we can see that while $t<3$ the error between the agents increases and once the controller is engaged after $t \geq 3$ the errors converge to zero; the rate of convergence depend on the nature of the operators.

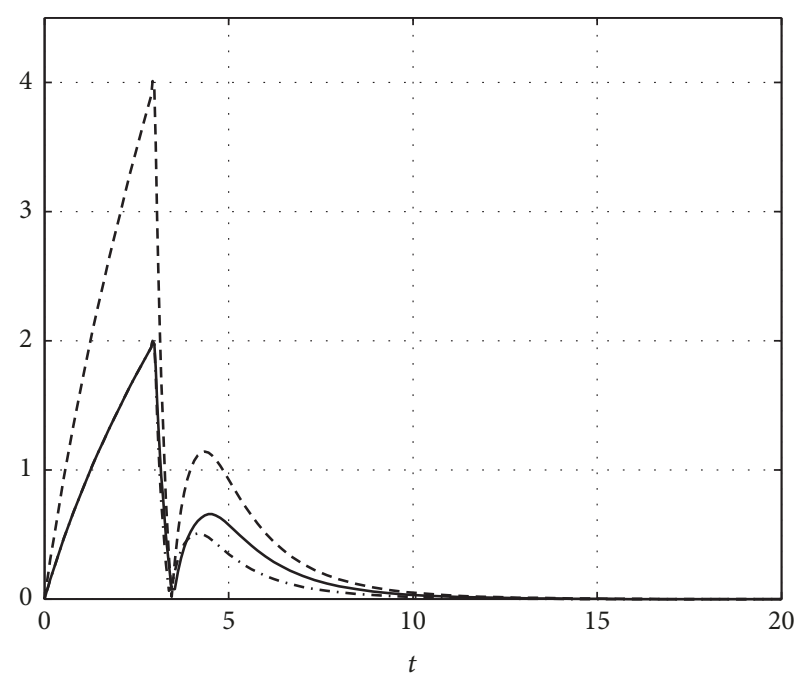

$-\left|x_{1}-x_{2}\right|$
$---\left|x_{2}-x_{3}\right|$
$\cdots-\left|x_{2}-x_{3}\right|$

FIgURE 12: No-linear case, $j=4$.

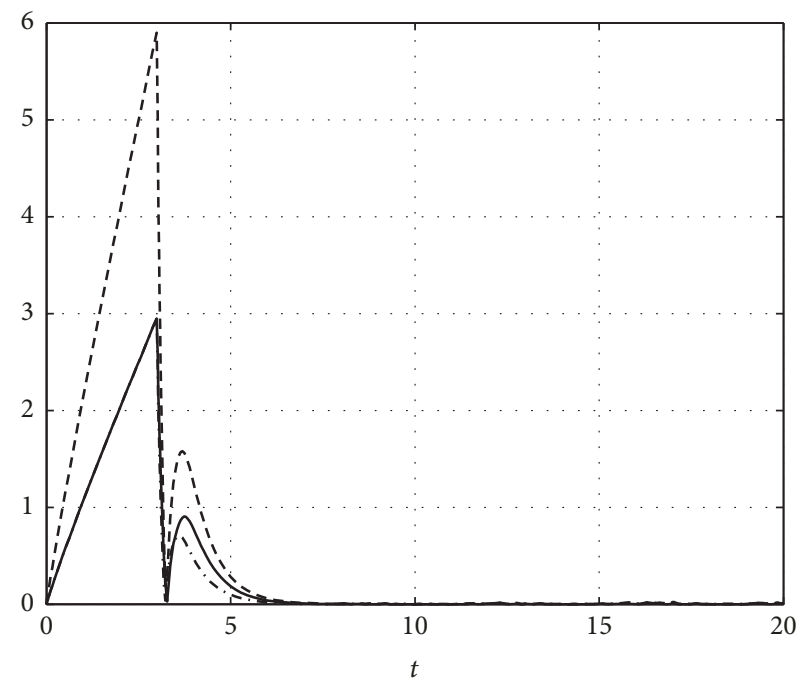

$-\left|x_{1}-x_{2}\right|$
$---\left|x_{2}-x_{3}\right|$
$-\cdot\left|x_{2}-x_{3}\right|$

FIGURE 13: No-linear case, $j=5$.

\section{Conclusions}

We introduced the distributed conformable derivative, which preserves the product and chain rules. For this and five other fractional derivatives, we unified the Lyapunov direct method. That result was presented in two theorems; the first bounds the Lyapunov function and its fractional derivative by powers of the norm of the states and the second by class $\mathscr{K}$ functions. Moreover, we employed this generalized fractional Lyapunov method to prove whether linear and nonlinear 


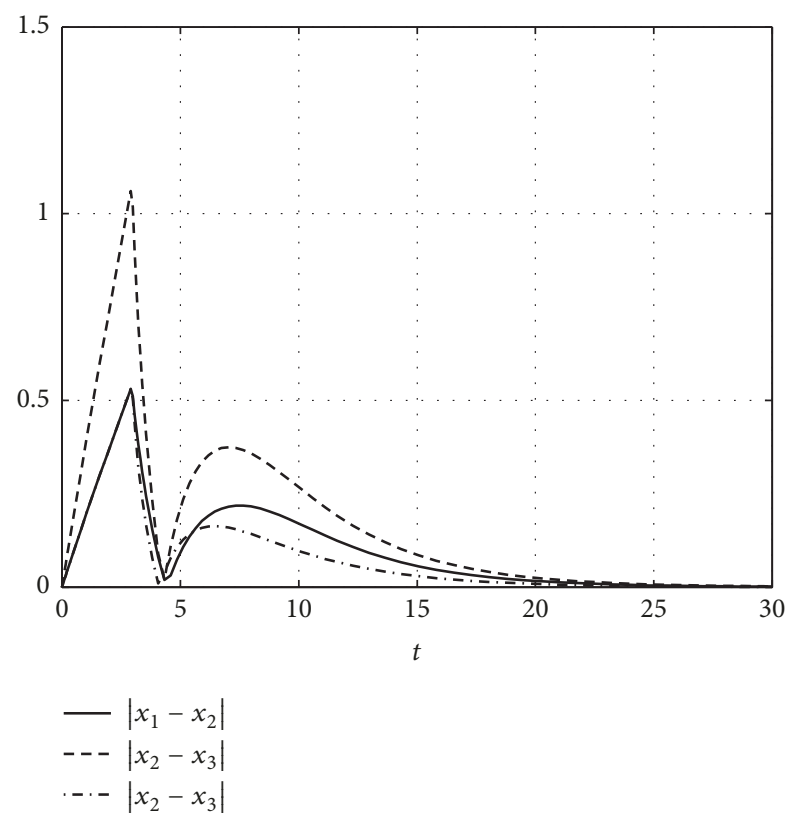

FIGURE 14: No-linear case, $j=6$.

multiagent systems, modeled with different fractional derivatives, accomplish consensus. We found that if the system is undisturbed, the agents converge asymptotically and if there are external disturbances, the steady-state errors evolve towards a region which diminishes linearly in size as the gain of the controller is increased. It is worth noticing that same control inputs are effective for all the differentiation orders considered in this paper.

In the light of these results, potential future objectives would be to carry out a similar analysis in the presence of time delays or to study the finite-time consensus problem for fractional multiagent systems, possibly employing different controllers.

\section{Data Availability}

The data used to support the findings of this study are available from the corresponding author upon request.

\section{Conflicts of Interest}

The authors declare that there are no conflicts of interest regarding the publication of this paper.

\section{Acknowledgments}

The financial support for this article is given through Universidad Iberoamericana Campus Ciudad de México and Universidad Católica del Uruguay as employers for the authors.

\section{References}

[1] G. W. F. Von Leibniz, Mathematische Schriften, vol. 1, Asher, 1849.

[2] A. Kilbas, H. M. Srivastava, and J. J. Trujillo, Theory and Applications of Fractional Differential Equations, New York, NY, USA, Elsevier, 2006.

[3] I. Petráš, Fractional-Order Nonlinear Systems: Modeling, Analysis and Simulation, Springer Science \& Business Media, 2011.

[4] I. Podlubny, Fractional Differential Equations, Academic Press, London, 1999.

[5] S. G. Samko and B. Ross, "Integration and differentiation to a variable fractional order," Integral Transforms and Special Functions, vol. 1, no. 4, pp. 277-300, 1993.

[6] H. G. Sun, W. Chen, H. Wei, and Y. Q. Chen, "A comparative study of constant-order and variable-order fractional models in characterizing memory property of systems," The European Physical Journal Special Topics, vol. 193, article no. 185, no. 1, 2011.

[7] M. Caputo, Elasticitá E Dissipazione, Zanichelli, Bologna, Italy, 1969.

[8] A. V. Chechkin, J. Klafter, and I. M. Sokolov, "Fractional FokkerPlanck equation for ultraslow kinetics," EPL (Europhysics Letters), vol. 63, no. 3, article no. 326, 2003.

[9] M. Naber, "Distributed order fractional sub-diffusion," Fractals, vol. 12, no. 1, pp. 23-32, 2004.

[10] C. F. Lorenzo and T. T. Hartley, "Variable order and distributed order fractional operators," Nonlinear Dynamics, vol. 29, no. 1-4, pp. 57-98, 2002.

[11] R. Khalil, M. Al Horani, A. Yousef, and M. Sababheh, "A new definition of fractional derivative," Journal of Computational and Applied Mathematics, vol. 264, pp. 65-70, 2014.

[12] R. Almeida, M. Guzowska, and T. Odzijewicz, "A remark on local fractional calculus and ordinary derivatives," Open Mathematics, vol. 14, pp. 1122-1124, 2016.

[13] N. Laskin, Fractional Quantum Mechanics, World Scientific, 2018.

[14] D. Baleanu, J. A. T. Machado, and A. C. J. Luo, Fractional Dynamics and Control, Springer, New York, NY, USA, 2012.

[15] S. S. Tabatabaei, M. J. Yazdanpanah, S. Jafari, and J. C. Sprott, "Extensions in dynamic models of happiness: Effect of memory," International Journal of Happiness and Development, vol. 1, no. 4, pp. 344-356, 2014.

[16] Y. Li, Y. Chen, and I. Podlubny, "Stability of fractional-order nonlinear dynamic systems: Lyapunov direct method and generalized Mittag-Leffler stability," Computers \& Mathematics with Applications, vol. 59, no. 5, pp. 1810-1821, 2010.

[17] A. Souahi, A. B. Makhlouf, and M. A. Hammami, "Stability analysis of conformable fractional-order nonlinear systems," Indagationes Mathematicae, vol. 28, no. 6, pp. 1265-1274, 2017.

[18] S. S. Tabatabaei, H. A. Talebi, and M. Tavakoli, "An adaptive order/state estimator for linear systems with non-integer timevarying order," Automatica, vol. 84, pp. 1-9, 2017.

[19] H. Taghavian and M. S. Tavazoei, "Stability analysis of distributed-order nonlinear dynamic systems," International Journal of Systems Science, vol. 49, no. 3, pp. 523-536, 2018.

[20] Y. Wang and T. Li, "Stability analysis of fractional-order nonlinear systems with delay," Mathematical Problems in Engineering, vol. 2014, Article ID 301235, 8 pages, 2014.

[21] W. Ren and R. W. Beard, Distributed Consensus in Multi-Vehicle Cooperative Control, Springer, 2008. 
[22] A. Jadbabaie, N. Motee, and M. Barahona, "On the stability of the Kuramoto model of coupled nonlinear oscillators," in Proceedings of the American Control Conference (AAC), pp. 4296-4301, IEEE, Boston, MA, USA, 2004.

[23] R. Olfati-Saber and J. S. Shamma, "Consensus filters for sensor networks and distributed sensor fusion," in Proceedings of the 44th IEEE Conference on Decision and Control, and the European Control Conference (CDC-ECC), pp. 6698-6703, IEEE, Seville, Spain, 2005.

[24] W. Ren and Y. Cao, Distributed Coordination of Multi-Agent Networks: Emergent Problems, Models, And Issues, Springer Science \& Business Media, 2010.

[25] Z. Yu, H. Jiang, C. Hu, and J. Yu, "Leader-following consensus of fractional-order multi-agent systems via adaptive pinning control," International Journal of Control, vol. 88, no. 9, pp. 17461756, 2015.

[26] X. Yin, D. Yue, and S. Hu, "Consensus of fractional-order heterogeneous multi-agent systems," IET Control Theory \& Applications, vol. 7, no. 2, pp. 314-322, 2013.

[27] C. Song, J. Cao, and Y. Liu, "Robust consensus of fractionalorder multi-agent systems with positive real uncertainty via second-order neighbors information," Neurocomputing, vol. 165, pp. 293-299, 2015.

[28] G. Nava-Antonio, G. Fernandez-Anaya, E. G. HernandezMartinez, J. Jamous-Galante, E. D. Ferreira-Vazquez, and J. J. Flores-Godoy, "Consensus of multi-agent systems with distributed fractional order dynamics," in Proceedings of the 14th International Workshop on Complex Systems and Networks (IWCSN), pp. 190-197, IEEE, Doha, Qatar, 2017.

[29] G. Ren and Y. Yu, "Robust consensus of fractional multi-agent systems with external disturbances," Neurocomputing, vol. 218, pp. 339-345, 2016.

[30] N. Aguila-Camacho, M. A. Duarte-Mermoud, and J. A. Gallegos, "Lyapunov functions for fractional order systems," Communications in Nonlinear Science and Numerical Simulation, vol. 19, no. 9, pp. 2951-2957, 2014.

[31] Z. Jiao, Y. Chen, and I. Podlubny, Distributed-Order Dynamic Systems Stability, Simulation, Applications and Perspectives, Springer Briefs in Electrical and Computer Engineering, Springer, 2012.

[32] Y. Xu and Z. He, "Existence and uniqueness results for Cauchy problem of variable-order fractional differential equations," Journal of Applied Mathematics and Computing, vol. 43, no. 12, pp. 295-306, 2013.

[33] N. J. Ford and M. L. Morgado, "Distributed order equations as boundary value problems," Computers \& Mathematics with Applications, vol. 64, no. 10, pp. 2973-2981, 2012.

[34] B. Bayour and D. F. M. Torres, "Existence of solution to a local fractional nonlinear differential equation," Journal of Computational and Applied Mathematics, vol. 312, pp. 127-133, 2017.

[35] D. G. Duffy, Transform Methods for Solving Partial Differential Equations, Symbolic \& Numeric Computation, CRC press, 2nd edition, 2004.

[36] A. R. Teel and L. Praly, "A smooth Lyapunov function from a class-KL estimate involving two positive semidefinite functions," ESAIM: Control, Optimisation and Calculus of Variations, vol. 5, pp. 313-367, 2000.

[37] G.-C. Wu, D. Baleanu, and W.-H. Luo, "Lyapunov functions for Riemann-Liouville-like fractional difference equations," Applied Mathematics and Computation, vol. 314, pp. 228-236, 2017.
[38] G. Fernández-Anaya, G. Nava-Antonio, J. Jamous-Galante, R. Muñoz-Vega, and E. G. Hernández-Martínez, "Asymptotic stability of distributed order nonlinear dynamical systems Asymptotic stability of distributed order nonlinear dynamical systems," Communications in Nonlinear Science and Numerical Simulation48541549, 2017.

[39] Y. Zhang and Y.-P. Tian, "Consentability and protocol design of multi-agent systems with stochastic switching topology," Automatica, vol. 45, no. 5, pp. 1195-1201, 2009.

[40] I. Petráš, "Fractional order chaotic systems," 2010, http://www .mathworks.com/matlabcentral/fileexchange/27336-fractionalorder-chaotic-systems.

[41] D. Valério, "Variable order derivatives," 2010, https://la.mathworks .com/matlabcentral/fileexchange/24444-variable-order-derivatives.

[42] D. Valério, G. Vinagre, J. Domingues, and J. S. Da Costa, "Variable-order fractional derivatives and their numerical approximations, I-Real orders," In Fractional Signals and Systems, 2009. 


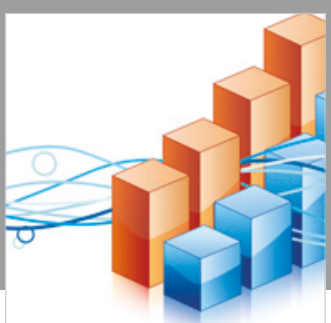

Advances in

Operations Research

\section{-n-m}
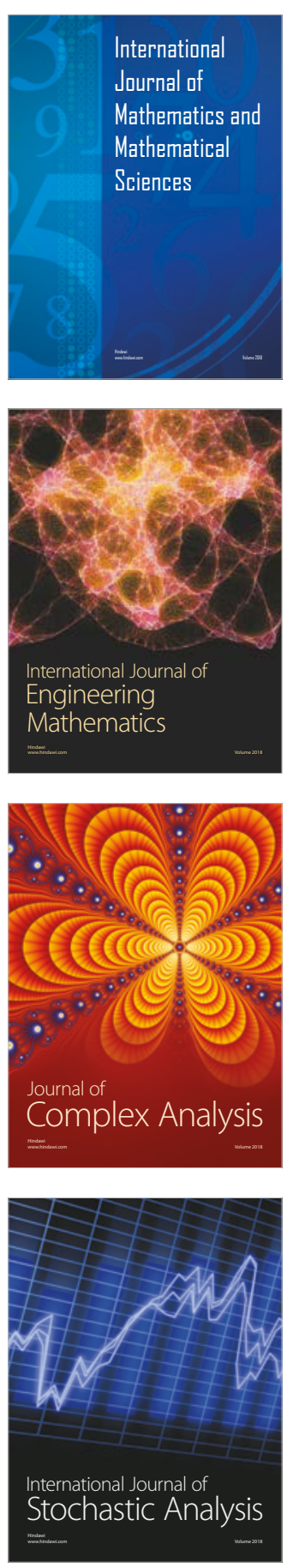
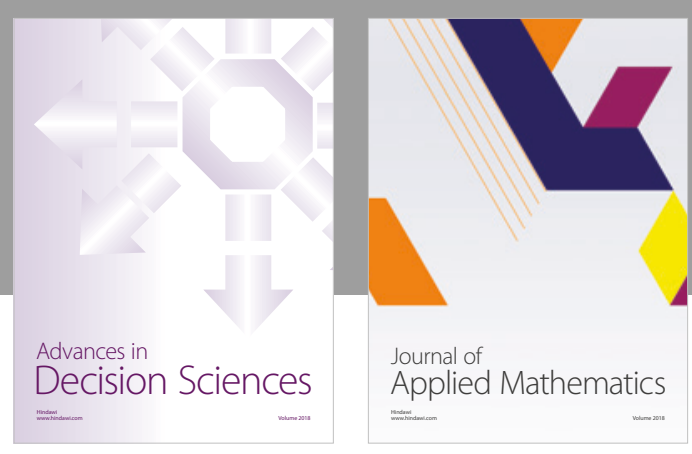

Journal of

Applied Mathematics
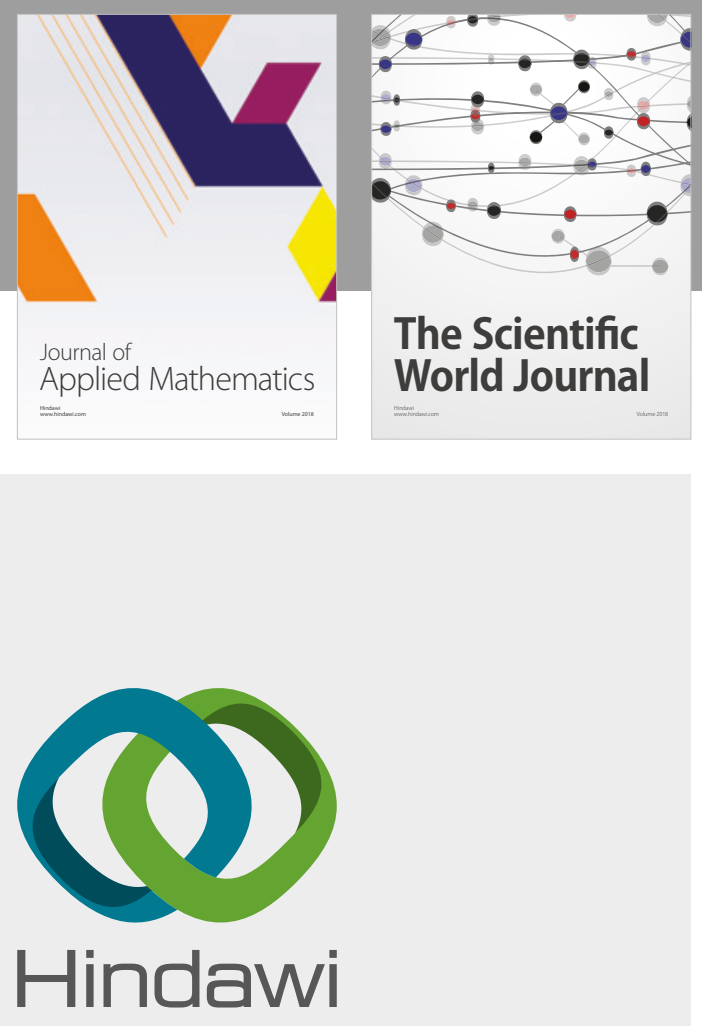

Submit your manuscripts at

www.hindawi.com

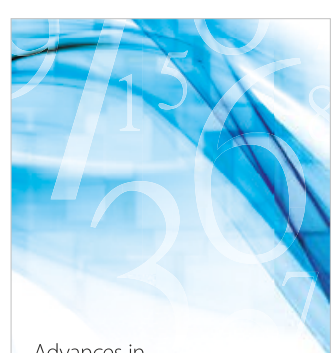

Advances in
Numerical Analysis
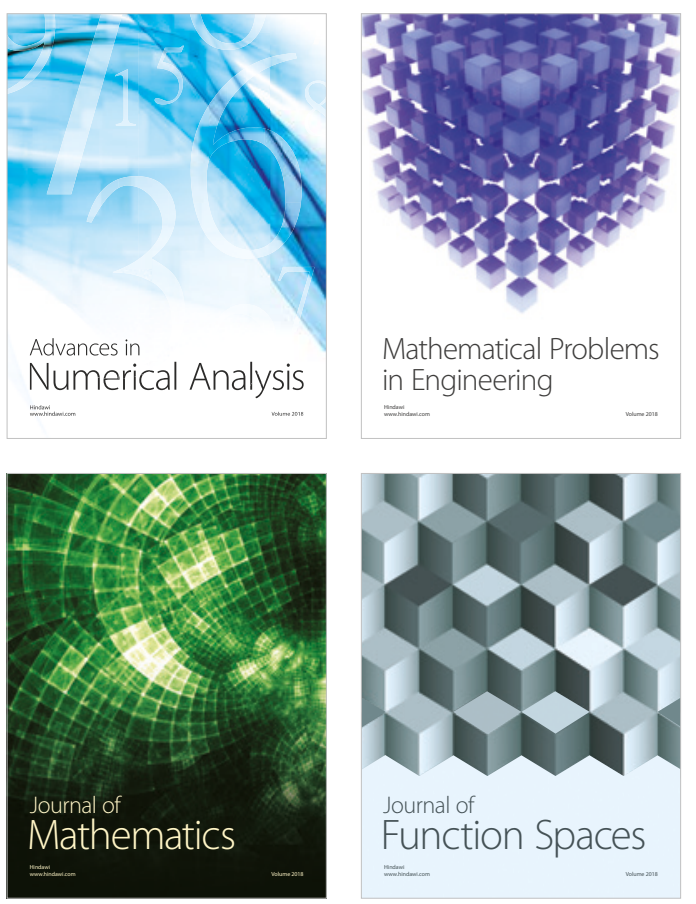

Mathematical Problems in Engineering

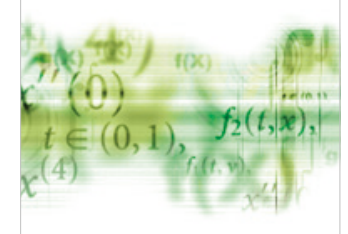

International Journal of

Differential Equations

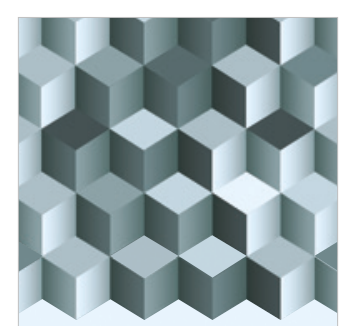

Journal of

Function Spaces
The Scientific

World Journal

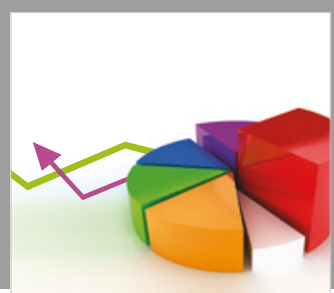

Journal of

Probability and Statistics
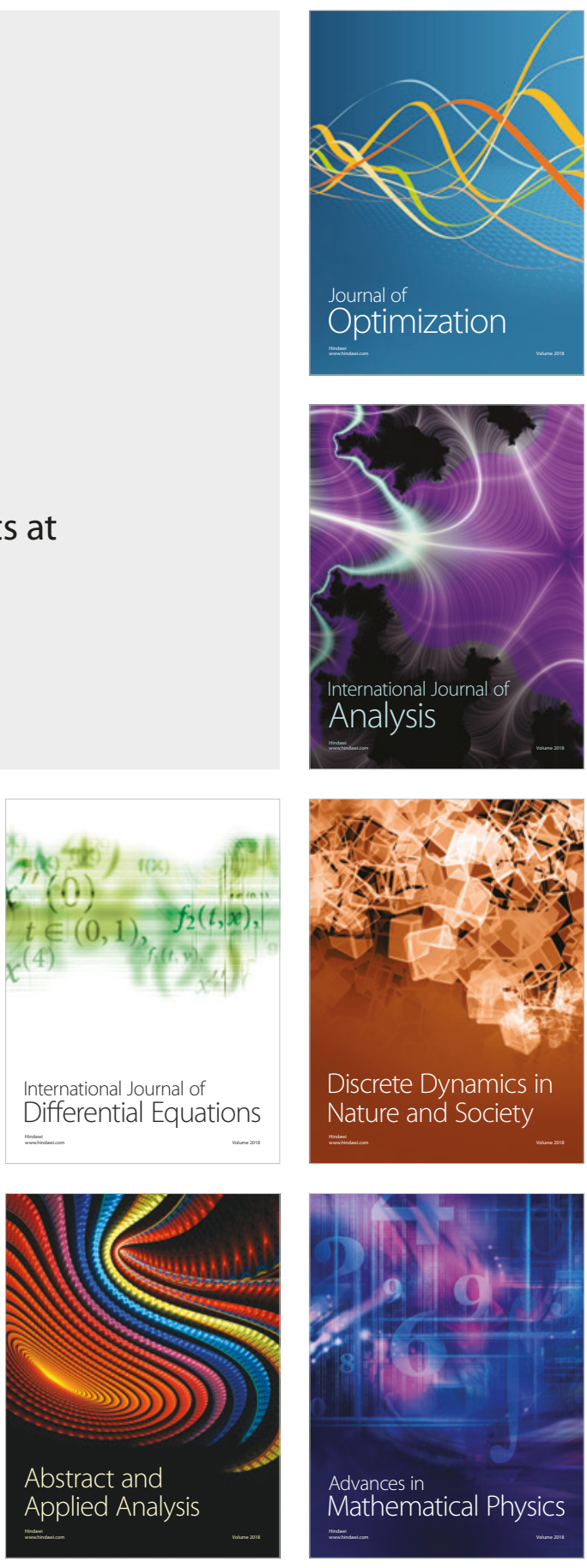